\title{
Entwicklung einer problemorientierten Wissensdatenbank für Pflegende
}

\author{
Anforderungen an eine evidenzbasierte Datenbank zu den Themengebieten Demenz \\ und Schmerz
}

\author{
Stefanie Schniering • Andreas Baumeister • \\ Ingrid Darmann-Finck $\cdot$ Stephan Zieme
}

Online publiziert: 30. Oktober 2013

(C) Springer-Verlag Wien 2013

\section{Development of a problem-oriented databank for nursing personnel}

Requirements for an evidence-based databank on the topics of dementia and pain

Hintergrund: Eine Möglichkeit, um den Transfer aktueller wissenschaftlicher Erkenntnisse in die Pflegepraxis zu unterstützen, stellen mediengestützte Lernangebote dar. Bislang werden die Potenziale mediengestützten Lernens für die Aus-, Fort- und Weiterbildung Pflegender aber kaum genutzt. Die Abteilung Qualifikations- und Curriculumforschung am Institut für Public Health und Pflegeforschung (IPP) der Universität Bremen und das Bremer Softwareunternehmen atacama | Software $\mathrm{GmbH}$ beabsichtigen daher die Entwicklung einer problemorientierten Wissensdatenbank für Pflegende, die einige Barrieren mediengestützter Wissensaneignung in der Pflege konstruktiv aufnimmt und den Pflegenden verständliches, evidenzbasiertes sowie bereits synthetisiertes Wissen zunächst zu den Themen „Demenz“ und „Schmerz“ zur Verfügung stellt. Im Mittelpunkt des Vortrags stehen die Methoden, Ergebnisse und die Diskussion ausgewählter Aspekte der Bedarfsanalyse der potenziellen Nutzer.
Ziel: Ziel der durchgeführten Bedarfsanalyse ist die Erfassung von Informationsbedürfnissen Pflegender zu den Themen Demenz und Schmerz sowie weiteren Anforderungen an eine Wissensdatenbank.

Methode: Als Forschungsdesign wurde ein qualitativer empirischer Zugang gewählt. Für die Datenerhebung wurden in den 5 beteiligten Piloteinrichtungen leitfadengestützte Interviews sowie „focus groups“ durchgeführt und anh and der qualitativen Inhaltsanalyse ausgewertet.

Ergebnis: Die Bedarfsanalyse kommt u. a. zu dem Ergebnis, dass die Befragten für die Pflegepraxis kurze komprimierte Informationen in Form von Kernaussagen und Handlungsanweisungen favorisieren. Zur Tiefe des Wissens bestehen unterschiedliche Präferenzen.

Schlussfolgerung: In der Diskussion wird festgestellt, dass die Datenbank tatsächlich geeignet ist, das mediengestützte Lernen zu forcieren, und problematisiert, dass die Wünsche der Pflegenden eher auf ein „regelorientiertes“ Pflegeverständnis schließen lassen. Aus den Ergebnissen werden im Anschluss Schlussfolgerungen für die Konzeption und Implementation der Datenbank gezogen.

S. Schniering · A. Baumeister · I. Darmann-Finck Institut für Public Health und Pflegeforschung (IPP), Abt. 4 Qualifikations- und Curriculumforschung,

Universität Bremen, Bremen, Deutschland

S. Zieme

atacama $\mid$ Software GmbH, Bremen, Deutschland 